\title{
和厚朴酚桥连卟啉光敏剂合成与结构表征
}

\author{
黄齐茂王司卫 邓鹏星周红胡学雷潘志权* \\ (武汉工程大学化工与制药学院 绿色化工过程省部共建教育部重点实验室 武汉 430073)
}

\begin{abstract}
摘要 依次利用 2-荎酚、1,6-二溴己烷、和厚朴酚，对 2-硝基-5,10,15,20-四苯基卟啉及其金属配合物进行结构修饰，首 次得到四对同分异构体桥连卟啉光敏剂, 目标产物经 UV-Vis, ${ }^{1} \mathrm{H}$ NMR, H-H COSY, IR, MS, 元素分析等表征. 以 1,3二苯基异苯并呋喃(DPBF)为猝灭剂测定了桥连卟啉产生单线态氧的能力; 采用凝胶电泳和紫外-可见光谱滴定法, 初 步考察了化合物对 pBR322 质粒 DNA 的光敏切割能力及其与小牛胸腺 DNA 的作用模式.

关键词 卟啉; 和厚朴酚; 表征; 光敏活性
\end{abstract}

\section{Synthesis and Characterization of Honokiol Bridged Porphyrins as Photosensitizers}

\author{
Huang, Qimao Wang, Siwei Deng, Pengxing Zhou, Hong \\ $\mathrm{Hu}$, Xuelei Pan, Zhiquan* \\ (Key Laboratory for Green Chemical Process of Ministry of Education, Wuhan Institute of Technology, Wuhan 430073)
}

\begin{abstract}
Four pairs of porphyrin isomers have been synthesized by reaction of 2-nitrotetraphenylporphyrin and its metallic complexes with 2-naphthol, 1,6-dibromohexane and honokiol respectively, and structurally characterized by UV-Vis, ${ }^{1} \mathrm{H}$ NMR, H-H COSY, IR, MS, and elemental analysis. The production of singlet oxygen under radiation was determined with 1,3-diphenylisobenzofuran (DPBF) as the quencher. Their photocleavage ability to pBR322 plasmid DNA has been tested by gel electrophoresis and the interaction with CT DNA was detected by UV-Vis spectroscopy preliminarily.
\end{abstract}

Keywords porphyrin; honokiol; characterization; photosensitivity

光动力治疗 (photodynamic therapy, PDT) 是一种创 伤小、疗效好的肿瘤临床治疗方法 ${ }^{[1]}$, 该疗法通过光敏 剂[2]选择性结合病变组织、在光照下产生活性氧杀灭癌 细胞而达到治疗目的, 高效光敏剂的选取是决定光动力 疗效的关键 ${ }^{[3]}$. 卟啉类化合物 ${ }^{[4]}$ 作为治疗恶性肿瘤的光 敏药物广泛应用于 PDT 领域, 其疗效基于卟啉化合物 对肿瘤组织的选择性富集及其光敏能力与药效, 官能团 修饰是改善其性能的重要手段, 其手段的多样性使卟啉 衍生物在 PDT 的应用前景更为广阔, 如设计合成同时 具有光动力效应和化学药效双重活性的抗癌小分子取 代的卟啉就是其中一类重要衍生化手段 ${ }^{[5]}$. 具有抗癌活 性的传统中药较多, 其有效成分中生物活性小分子纯品 商品化也有案例, 如天然产物和厚朴酚 (honokiol) 是从 木兰科植物厚朴的根皮和枝皮中分离提取的主要活性
成分之一，具有广谱抗菌、抗炎、抗病毒、抗肿瘤、抗 氧化和抗衰老等药理作用 ${ }^{[6]}$. 本文在原有工作基础上 ${ }^{[7]}$ 将该中药活性小分子与特定卟啉桥连，合成得到和厚朴 酚桥连卟啉衍生物(Scheme 1). 这是首次将和厚朴酚引 入光动力领域, 该新型光敏剂的设计是期望通过可能的 光动力效应和化学药效协同作用 ${ }^{[8]}$ 达到增强抗癌疗效的 目的，在结构表征基础上的初步光敏活性研究表明，新 型同分异构体光敏剂具有良好的产单线态氧能力，对 DNA 光敏切割效果明显, 且与 DNA 结合能力较强.

\section{1 实验部分}

\section{1 仪器与试剂}

Shimadzu UV-2450 紫外-可见分光光度计; VarianMercury 300 超导核磁共振仪; Shimadzu FTIR-8100 红外

\footnotetext{
*E-mail: zhiqpan@163.com or huangqim@163.com; Tel.: 027-87194980.

Received October 12, 2011; revised February 16, 2012; published online March 22, 2012.

Project supported by the National Natural Science Foundation of China (No. 20471045), the Natural Science Foundation of Hubei Province (No. 2008CDB072), the Foundation for Midlife and Youth Excellent Innovation Group of Hubei Province (2008).

国家自然科学基金(No. 20471045)、湖北省自然科学基金青年杰出人才(No. 2008CDB072)、湖北省高等学校优秀中青年团队计划(2008)资助项目.
} 
光谱仪; Varian QFT-ESI 傅立叶变换离子回旋共振质谱 仪; Perkin-Elmer 204B 元素分析仪; 高压录灯波长范围 350 800 nm, $50 \mathrm{~W}$; DYY-8B 稳流稳压电泳仪; Vilber Lourmat Bio Print-1000 凝胶成像分析系统.

所有化学试剂均用标准方法纯化. 柱层析硅胶粒度 200 300 目, 薄层层析硅胶型号 GF254; 和厚朴酚购自 西安旭煌公司; 三差弪甲基氨基甲烷-盐酸(Tris-HCl)缓冲 液, 琼脂糖, TOYOBO pBR322 质粒 DNA, 1,3-二苯基异 苯并呋喃(DPBF), 小牛胸腺 DNA (CT DNA), 澳酚蓝, 澳化乙锭(EB)均购自武汉华美公司.

2-硝基 -5,10,15,20-四苯基卟啉，2-(2-羟基萗 基)-5,10,15,20-四苯基卟啉，2-[2-(6-溴)已氧基]荎基5,10,15,20-四苯基卟啉及其 $\mathrm{Cu}(\mathrm{II}), \mathrm{Ni}(\mathrm{II}), \mathrm{Zn}(\mathrm{II})$ 配合物 1 4 的合成参考文献[9].

\section{2 和厚朴酚桥连卟啉铜 5 和 9 的合成}

称取 $400 \mathrm{mg}(0.4 \mathrm{mmol})$ 的 2-[2-(6-溴)己氧基]菜基-
5,10,15,20-四苯基卟啉铜(1)用 $100 \mathrm{~mL}$ 无水 $\mathrm{DMF}$ 溶于 $250 \mathrm{~mL}$ 三口烧瓶中, 电磁搅拌至卟啉完全溶解, 分别加 入 $1.5 \mathrm{~g}$ 刚焙烧过的无水碳酸钾和 $160 \mathrm{mg}(0.6 \mathrm{mmol})$ 和 厚朴酚，于 $35^{\circ} \mathrm{C}$ 下反应 $5 \mathrm{~h}$. 反应完毕后加入 $200 \mathrm{~mL}$ 蒸 馏水，抽滤，滤饼烘干后以硅胶(200 300 目)为固定相, 混合溶液 $(V: V=1: 1)$ 为淋洗剂, 进行柱层析分离, 分 别收集, 浓缩至饱和, 加入热乙醇重结晶, 得红色晶体 $5154 \mathrm{mg}$ (0.13 mmol), 产率约 33\%; 红色晶体 $9143 \mathrm{mg}$ $(0.12 \mathrm{mmol})$, 产率约 $30 \%$.

5: m.p. $>300{ }^{\circ} \mathrm{C}$; UV-Vis (DMF) $\lambda_{\max }: 417,540 \mathrm{~nm}$; IR (KBr) v: 3434 (OH), 2926, 2858 (ArC-H), 1596, 1490 $\mathrm{ArH}), 1266(\mathrm{C}-\mathrm{O}-\mathrm{C}), 1170(\mathrm{C}-\mathrm{O}), 1004(\mathrm{~N}-\mathrm{Cu})$, 799, 749, $701\left(\mathrm{CH}_{2}\right) \mathrm{cm}^{-1}$; MS (QFT-ESI) $\mathrm{m} / \mathrm{z}(\%)$ : $1188.4526\left([\mathrm{M}+\mathrm{Na}]^{+}, 100\right)$. Anal. calcd for $\mathrm{C}_{78} \mathrm{H}_{62} \mathrm{~N}_{4^{-}}$ $\mathrm{O}_{3} \mathrm{Cu}: \mathrm{C} 80.28, \mathrm{H} 5.36, \mathrm{~N} 4.80$; found C 80.56, H 5.49, N 4.74.

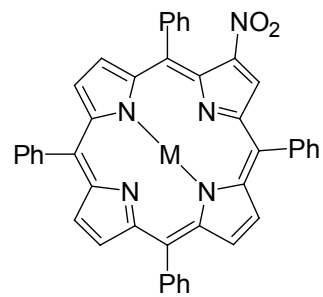<smiles>O=[N+]([O-])OCCOc1ccc2ccccc2c1</smiles>

$\mathrm{NaOH} / \mathrm{DMF}$

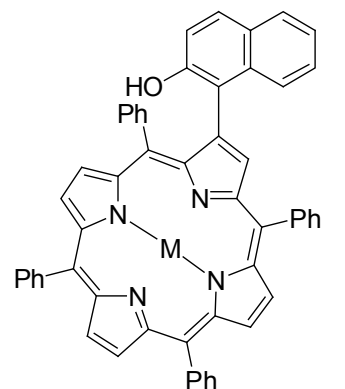

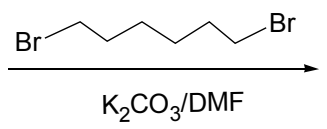

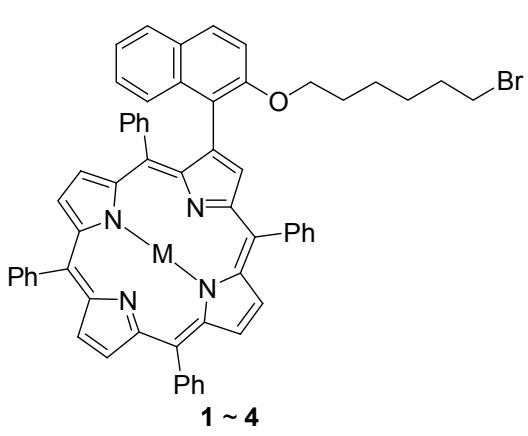

1: $M=C u ; 2: M=N i ; 3: M=Z n ; 4: M=2 H$

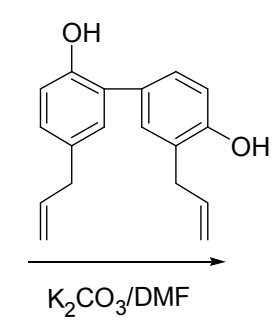

Ph
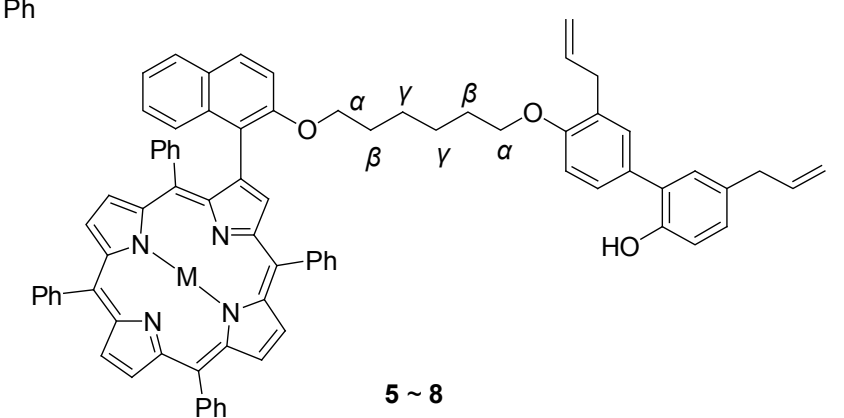

5: $M=C u ; 6: M=N i ;: M=Z n ; 8: M=2 H$

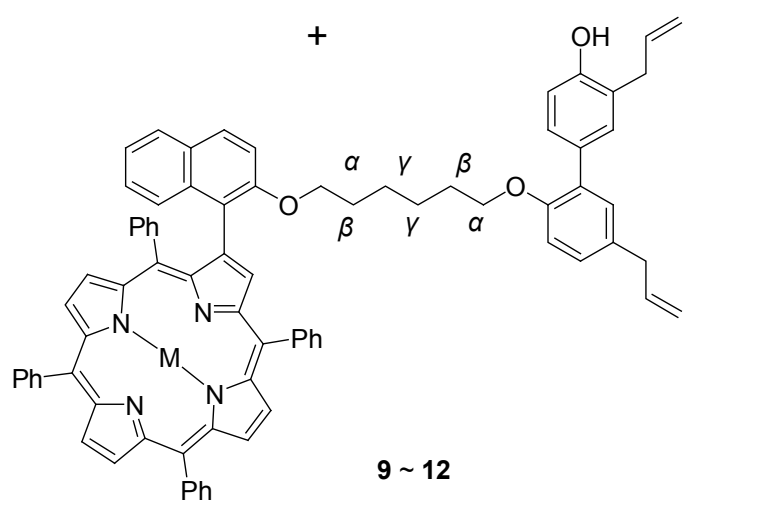

9: $M=C u ; 10: M=N i ; 11: M=Z n ; 12: M=2 H$

Scheme 1 
9: m.p. $>300{ }^{\circ} \mathrm{C}$; UV-Vis (DMF) $\lambda_{\max }: 417,540 \mathrm{~nm}$; IR (KBr) v: $3433(\mathrm{OH}), 2924,2855(\mathrm{ArC}-\mathrm{H}), 1596,1490$ $(\mathrm{ArH}), 1266(\mathrm{C}-\mathrm{O}-\mathrm{C}), 1178(\mathrm{C}-\mathrm{O}), 1004(\mathrm{~N}-\mathrm{Cu})$, 799, 749, $701\left(\mathrm{CH}_{2}\right) \mathrm{cm}^{-1}$; MS (QFT-ESI) $\mathrm{m} / \mathrm{z}(\%)$ : $1166.4269\left([\mathrm{M}+\mathrm{H}]^{+}, 100\right)$. Anal. calcd for $\mathrm{C}_{78} \mathrm{H}_{62} \mathrm{~N}_{4} \mathrm{O}_{3^{-}}$ $\mathrm{Cu}$ : C 80.28, H 5.36, N 4.80; found C 80.49, H 5.42, N 4.65 .

\section{3 和厚朴酚桥连卟啉镍 6 和 10 的合成}

称取 $400 \mathrm{mg}(0.4 \mathrm{mmol})$ 的 2-[2-(6-溴)己氧基]萗基5,10,15,20-四苯基卟啉镍(2)用 $100 \mathrm{~mL}$ 无水 $\mathrm{DMF}$ 溶于 $250 \mathrm{~mL}$ 三口烧瓶中, 其它操作同上, 得鲜红色晶体 $\mathbf{6}$ $148 \mathrm{mg}(0.13 \mathrm{mmol})$, 产率约 $32 \%$; 鲜红色晶体 10152 $\mathrm{mg}(0.13 \mathrm{mmol})$, 产率约 $33 \%$.

6: m.p. $>300{ }^{\circ} \mathrm{C}$; UV-Vis (DMF) $\lambda_{\max }: 417,531 \mathrm{~nm}$; ${ }^{1} \mathrm{H}$ NMR $\left(\mathrm{CDCl}_{3}, 300 \mathrm{MHz}\right) \delta: 8.74 \sim 8.38(\mathrm{~m}, 7 \mathrm{H}$, $\beta$-pyrrole-H), $8.01 \sim 7.39(\mathrm{~m}, 20 \mathrm{H}, \quad 5,10,15,20-\mathrm{Ph}-\mathrm{H})$, $7.36 \sim 6.34\left(\mathrm{~m}, 12 \mathrm{H}, 2-3^{\prime} 4^{\prime} 5^{\prime} 6^{\prime} 7^{\prime} 8^{\prime}-\mathrm{H}, \mathrm{ArH}\right), 6.07(\mathrm{~s}, 1 \mathrm{H}$, $\mathrm{OH}), 5.93 \sim 5.86(\mathrm{~m}, 2 \mathrm{H}, \mathrm{CH}=), 5.16 \sim 4.83(\mathrm{~m}, 4 \mathrm{H}, \mathrm{C}=$ $\left.\mathrm{CH}_{2}\right), 3.89 \sim 2.88\left(\mathrm{~m}, 8 \mathrm{H}\right.$, aryl- $\left.\mathrm{CH}_{2}, \alpha-\mathrm{CH}_{2}\right), 1.39 \sim 1.26$ (m, $\left.4 \mathrm{H}, \beta-\mathrm{CH}_{2}\right), 0.86 \sim 0.81\left(\mathrm{~m}, 4 \mathrm{H}, \gamma-\mathrm{CH}_{2}\right)$; IR (KBr) $v$ : $3435(\mathrm{OH}), 2925,2853(\mathrm{ArC}-\mathrm{H}), 1598,1492(\mathrm{ArH})$, $1245(\mathrm{C}-\mathrm{O}-\mathrm{C}), 1178 \quad(\mathrm{C}-\mathrm{O}), 1009(\mathrm{~N}-\mathrm{Ni}), 798$, 750, $701\left(\mathrm{CH}_{2}\right) \mathrm{cm}^{-1}$; MS (QFT-ESI) $\mathrm{m} / z(\%): 1183.4062$ $\left([\mathrm{M}+\mathrm{Na}]^{+}, 100\right)$. Anal. calcd for $\mathrm{C}_{78} \mathrm{H}_{62} \mathrm{~N}_{4} \mathrm{O}_{3} \mathrm{Ni}$ : C 80.62, $\mathrm{H}$ 5.38, N 4.82; found C 80.89, H 5.52, N 4.77.

10: m.p. $>300{ }^{\circ} \mathrm{C}$; UV-Vis (DMF) $\lambda_{\max }: 417,531$ $\mathrm{nm} ;{ }^{1} \mathrm{H}$ NMR $\left(\mathrm{CDCl}_{3}, 300 \mathrm{MHz}\right) \delta: 8.75 \sim 8.37(\mathrm{~m}, 7 \mathrm{H}$, $\beta$-pyrrole-H), $8.02 \sim 7.42(\mathrm{~m}, 20 \mathrm{H}, 5,10,15,20-\mathrm{Ph}-\mathrm{H})$, $7.35 \sim 6.26\left(\mathrm{~m}, 12 \mathrm{H}, 2-3^{\prime} 4^{\prime} 5^{\prime} 6^{\prime} 7^{\prime} 8^{\prime}-\mathrm{H}, \mathrm{ArH}\right), 6.16(\mathrm{~s}, 1 \mathrm{H}$, $\mathrm{OH}), 5.89 \sim 5.75(\mathrm{~m}, 2 \mathrm{H}, \mathrm{CH}=), 5.06 \sim 4.82(\mathrm{~m}, 4 \mathrm{H}, \mathrm{C}=$ $\left.\mathrm{CH}_{2}\right), 3.86 \sim 2.87\left(\mathrm{~m}, 8 \mathrm{H}\right.$, aryl- $\left.\mathrm{CH}_{2}, \alpha-\mathrm{CH}_{2}\right), 1.34 \sim 1.24$ (m, $\left.4 \mathrm{H}, \beta-\mathrm{CH}_{2}\right), 0.83 \sim 0.77\left(\mathrm{~m}, 4 \mathrm{H}, \gamma-\mathrm{CH}_{2}\right)$; IR (KBr) $v$ : $3433(\mathrm{OH}), 2923,2850(\mathrm{ArC}-\mathrm{H}), 1597,1491(\mathrm{ArH})$, $1238(\mathrm{C}-\mathrm{O}-\mathrm{C}), 1144(\mathrm{C}-\mathrm{O}), 1008(\mathrm{~N}-\mathrm{Ni}), 797,750$, $701\left(\mathrm{CH}_{2}\right) \mathrm{cm}^{-1}$; MS (QFT-ESI) $\mathrm{m} / \mathrm{z}(\%): 1161.4253$ $\left([\mathrm{M}+\mathrm{H}]^{+}, 100\right)$. Anal. calcd for $\mathrm{C}_{78} \mathrm{H}_{62} \mathrm{~N}_{4} \mathrm{O}_{3} \mathrm{Ni}$ : C 80.62, H 5.38, N 4.82; found C 80.96, H 5.45, N 4.76.

\section{4 和厚朴酚桥连卟啉锌 7 和 11 的合成}

称取 $400 \mathrm{mg}(0.4 \mathrm{mmol})$ 的 2-[2-(6-溴)己氧基]荟基5,10,15,20-四苯基卟啉锌(3)用 $100 \mathrm{~mL}$ 无水 $\mathrm{DMF}$ 溶于 $250 \mathrm{~mL}$ 三口烧瓶中, 其它操作同上, 得亮紫色晶体 7 $156 \mathrm{mg}$ (0.13 mmol), 产率约 33\%; 亮紫色晶体 11151 $\mathrm{mg}(0.13 \mathrm{mmol})$, 产率约 $33 \%$.

7: m.p. $>300{ }^{\circ} \mathrm{C}$; UV-Vis (DMF) $\lambda_{\text {max }}: 427,560 \mathrm{~nm}$; ${ }^{1} \mathrm{H}$ NMR $\left(\mathrm{CDCl}_{3}, 300 \mathrm{MHz}\right) \delta: 8.74 \sim 8.38(\mathrm{~m}, 7 \mathrm{H}$,
$\beta$-pyrrole-H), $8.01 \sim 7.39(\mathrm{~m}, \quad 20 \mathrm{H}, \quad 5,10,15,20-\mathrm{Ph}-\mathrm{H})$, $7.36 \sim 6.34\left(\mathrm{~m}, 12 \mathrm{H}, 2-3^{\prime} 4^{\prime} 5^{\prime} 6^{\prime} 7^{\prime} 8^{\prime}-\mathrm{H}, \operatorname{ArH}\right), 6.07(\mathrm{~s}, 1 \mathrm{H}$, $\mathrm{OH}), 5.93 \sim 5.86(\mathrm{~m}, 2 \mathrm{H}, \mathrm{CH}=), 5.16 \sim 4.83(\mathrm{~m}, 4 \mathrm{H}, \mathrm{C}=$ $\left.\mathrm{CH}_{2}\right), 3.89 \sim 2.88\left(\mathrm{~m}, 8 \mathrm{H}\right.$, aryl- $\left.\mathrm{CH}_{2}, \alpha-\mathrm{CH}_{2}\right), 1.39 \sim 1.26$ (m, $4 \mathrm{H}, \beta-\mathrm{CH}_{2}$ ), $0.86 \sim 0.81$ (m, $4 \mathrm{H}, \gamma-\mathrm{CH}_{2}$ ); IR (KBr) $v$ : $3435(\mathrm{OH}), 2922,2852(\mathrm{ArC}-\mathrm{H}), 1596,1488(\mathrm{ArH})$, $1243(\mathrm{C}-\mathrm{O}-\mathrm{C}), 1178(\mathrm{C}-\mathrm{O}), 1000(\mathrm{~N}-\mathrm{Zn}), 797,748$, $700\left(\mathrm{CH}_{2}\right) \mathrm{cm}^{-1}$; MS (QFT-ESI) $\mathrm{m} / z(\%): 1189.4316$ $\left([\mathrm{M}+\mathrm{Na}]^{+}, 100\right)$. Anal. calcd for $\mathrm{C}_{78} \mathrm{H}_{62} \mathrm{~N}_{4} \mathrm{O}_{3} \mathrm{Zn}: \mathrm{C} 80.16$, H 5.35, N 4.79; found C 80.39, H 5.43, N 4.71.

11: m.p. $>300{ }^{\circ} \mathrm{C}$; UV-Vis (DMF) $\lambda_{\max }: 427,560 \mathrm{~nm}$; ${ }^{1} \mathrm{H}$ NMR $\left(\mathrm{CDCl}_{3}, 300 \mathrm{MHz}\right) \delta: 8.75 \sim 8.37(\mathrm{~m}, 7 \mathrm{H}$, $\beta$-pyrrole-H), $8.02 \sim 7.42(\mathrm{~m}, 20 \mathrm{H}, 5,10,15,20-\mathrm{Ph}-\mathrm{H})$, $7.35 \sim 6.26\left(\mathrm{~m}, 12 \mathrm{H}, 2-3^{\prime} 4^{\prime} 5^{\prime} 6^{\prime} 7^{\prime} 8^{\prime}-\mathrm{H}, \mathrm{ArH}\right), 6.16(\mathrm{~s}, 1 \mathrm{H}$, $\mathrm{OH}), 5.89 \sim 5.75(\mathrm{~m}, 2 \mathrm{H}, \mathrm{CH}=), 5.06 \sim 4.82(\mathrm{~m}, 4 \mathrm{H}, \mathrm{C}=$ $\left.\mathrm{CH}_{2}\right), 3.86 \sim 2.87\left(\mathrm{~m}, 8 \mathrm{H}\right.$, aryl- $\left.\mathrm{CH}_{2}, \alpha-\mathrm{CH}_{2}\right), 1.34 \sim 1.24$ (m, $4 \mathrm{H}, \beta-\mathrm{CH}_{2}$ ), $0.83 \sim 0.77$ (m, $4 \mathrm{H}, \gamma-\mathrm{CH}_{2}$ ); IR (KBr) $v$ : $3434(\mathrm{OH}), 2922,2852(\mathrm{ArC}-\mathrm{H}), 1595,1487$ (ArH), $1243(\mathrm{C}-\mathrm{O}-\mathrm{C}), 1175(\mathrm{C}-\mathrm{O}), 1000(\mathrm{~N}-\mathrm{Zn}), 796,746$, $700\left(\mathrm{CH}_{2}\right) \mathrm{cm}^{-1}$; MS (QFT-ESI) $\mathrm{m} / \mathrm{z}$ (\%): 1167.4207 $\left([\mathrm{M}+\mathrm{H}]^{+}, 100\right)$. Anal. calcd for $\mathrm{C}_{78} \mathrm{H}_{62} \mathrm{~N}_{4} \mathrm{O}_{3} \mathrm{Zn}: \mathrm{C} 80.16$, H 5.35, N 4.79; found C 80.41, H 5.44, N 4.67.

\section{5 和厚朴酚桥连卟啉 8 和 12 的合成}

称取 $400 \mathrm{mg}(0.4 \mathrm{mmol})$ 的 2-[2-(6-溴)己氧基]荎基5,10,15,20-四苯基卟啉(4)用 $100 \mathrm{~mL}$ 无水 DMF 溶于 250 $\mathrm{mL}$ 三口烧瓶中, 其它操作同上, 得紫色晶体 $8139 \mathrm{mg}$ (0.12 mmol), 产率约 32\%; 紫色晶体 $12144 \mathrm{mg}$ (0.13 $\mathrm{mmol})$, 产率约 $33 \%$.

8: m.p. $>300{ }^{\circ} \mathrm{C}$; UV-Vis (DMF) $\lambda_{\max }: 418,516 \mathrm{~nm}$; ${ }^{1} \mathrm{H}$ NMR $\left(\mathrm{CDCl}_{3}, 300 \mathrm{MHz}\right) \delta: 8.79 \sim 8.48(\mathrm{~m}, 7 \mathrm{H}$, $\beta$-pyrrole-H), $8.27 \sim 7.39(\mathrm{~m}, 20 \mathrm{H}, \quad 5,10,15,20-\mathrm{Ph}-\mathrm{H})$, $7.28 \sim 6.21\left(\mathrm{~m}, 12 \mathrm{H}, 2-3^{\prime} 4^{\prime} 5^{\prime} 6^{\prime} 7^{\prime} 8^{\prime}-\mathrm{H}, \mathrm{ArH}\right), 6.01(\mathrm{~s}, 1 \mathrm{H}$, $\mathrm{OH}), 5.92 \sim 5.83(\mathrm{~m}, 2 \mathrm{H}, \mathrm{CH}=), 5.17 \sim 4.84(\mathrm{~m}, 4 \mathrm{H}, \mathrm{C}=$ $\mathrm{CH}_{2}$ ), $3.87 \sim 2.86\left(\mathrm{~m}, 8 \mathrm{H}\right.$, aryl- $\left.\mathrm{CH}_{2}, \alpha-\mathrm{CH}_{2}\right), 1.41 \sim 1.25$ $\left(\mathrm{m}, 4 \mathrm{H}, \beta-\mathrm{CH}_{2}\right), 0.89 \sim 0.78\left(\mathrm{~m}, 4 \mathrm{H}, \gamma-\mathrm{CH}_{2}\right),-2.59(\mathrm{~s}$, 2H, NH); IR (KBr) v: $3434(\mathrm{OH}), 2924,2854(\mathrm{ArC}-\mathrm{H})$, 1597, 1492 (ArH), $1244 \quad(\mathrm{C}-\mathrm{O}-\mathrm{C}), 1177(\mathrm{C}-\mathrm{O}), 965$ $(\mathrm{N}-\mathrm{H}), 801,731,701\left(\mathrm{CH}_{2}\right) \mathrm{cm}^{-1}$; MS (QFT-ESI) $\mathrm{m} / \mathrm{z}$ (\%): $1127.4963\left([\mathrm{M}+\mathrm{Na}]^{+}, 100\right)$. Anal. calcd for $\mathrm{C}_{78} \mathrm{H}_{64}-$ $\mathrm{N}_{4} \mathrm{O}_{3}$ : C 84.75, H 5.84, N 5.07; found C 84.93, H 5.96, N 4.92 .

12: m.p. $>300{ }^{\circ} \mathrm{C}$; UV-Vis (DMF) $\lambda_{\max }: 418,516 \mathrm{~nm}$; ${ }^{1} \mathrm{H}$ NMR $\left(\mathrm{CDCl}_{3}, 300 \mathrm{MHz}\right) \delta: 8.81 \sim 8.63(\mathrm{~m}, 7 \mathrm{H}$, $\beta$-pyrrole-H), $8.28 \sim 7.41(\mathrm{~m}, 20 \mathrm{H}, \quad 5,10,15,20-\mathrm{Ph}-\mathrm{H})$, 7.26 6.18 (m, 12H, 2-3'4'5' $\left.6^{\prime} 7^{\prime} 8^{\prime}-\mathrm{H}, \mathrm{ArH}\right), 6.09$ (s, 1H, 
$\mathrm{OH}), 5.87 \sim 5.74(\mathrm{~m}, 2 \mathrm{H}, \mathrm{CH}=), 5.13 \sim 4.82(\mathrm{~m}, 4 \mathrm{H}, \mathrm{C}=$ $\left.\mathrm{CH}_{2}\right), 3.86 \sim 2.87\left(\mathrm{~m}, 8 \mathrm{H}\right.$, aryl- $\left.\mathrm{CH}_{2}, \alpha-\mathrm{CH}_{2}\right), 1.39 \sim 1.26$ $\left(\mathrm{m}, 4 \mathrm{H}, \beta-\mathrm{CH}_{2}\right), 0.87 \sim 0.79\left(\mathrm{~m}, 4 \mathrm{H}, \gamma-\mathrm{CH}_{2}\right),-2.61(\mathrm{~s}$, 2H, NH); IR (KBr) v: $3433(\mathrm{OH}), 2924,2854(\mathrm{ArC}-\mathrm{H})$, 1596, 1492 (ArH), $1244(\mathrm{C}-\mathrm{O}-\mathrm{C}), 1177(\mathrm{C}-\mathrm{O}), 965$ $(\mathrm{N}-\mathrm{H}), 801,731,701\left(\mathrm{CH}_{2}\right) \mathrm{cm}^{-1}$; MS (QFT-ESI) $\mathrm{m} / \mathrm{z}$ (\%): $1105.5811\left([\mathrm{M}+\mathrm{H}]^{+}, 100\right)$. Anal. calcd for $\mathrm{C}_{78} \mathrm{H}_{64}-$ $\mathrm{N}_{4} \mathrm{O}_{3}$ : C 84.75, H 5.84, N 5.07; found C 84.98, H 5.92, N 4.89.

\section{6 卟啉产生单线态氧能力的测定}

将卟啉溶液和 DPBF 混溶于 Tris-HCl 缓冲液中, 使 卟啉浓度为 $1 \mu \mathrm{mol} / \mathrm{L}, \mathrm{DPBF}$ 浓度为 $100 \mu \mathrm{mol} / \mathrm{L}$. 取 3 $\mathrm{mL}$ 上述溶液于比色血中, 以高压永灯照射(距样品 20 $\mathrm{cm}$ ), 用紫外分光光度计测量随时间变化的溶液在 415 $\mathrm{nm}$ 处吸光度值, 以光照时间为横坐标与相应吸光度为 纵坐标作图, 测定其产生单线态氧的能力.

\section{7 卟啉切割 $\mathrm{pBR} 322$ 质粒 DNA 的测定}

$1 \%$ 的琼脂糖(含 $0.1 \%$ 的 $\mathrm{EB}$ )制成凝胶. pBR322 质粒 DNA $(1.0 \mu \mathrm{g})$ 分别和不同浓度的卟啉混合使其总体积为 $10 \mu \mathrm{L}$, 于 $37{ }^{\circ} \mathrm{C}$ 下用高压录灯照射(距离为 $20 \mathrm{~cm}$ ), 同时 作未光照的对照实验. 停止光照后加 $1 \mu \mathrm{L}$ 澳酚蓝, 在电 压为 $100 \mathrm{~V}$ 下电泳 $1.5 \mathrm{~h}$ 后, 用凝胶成像系统观察结果并 记录.

\section{8 卟啉与 CT DNA 紫外-可见光谱滴定}

卟啉浓度为 $1 \mu \mathrm{mol} / \mathrm{L}$, 在紫外-可见光谱仪的参比 池和样品池中各加入 $3.0 \mathrm{~mL}$ Tris- $\mathrm{HCl}$ 缓冲溶液, 样品池 加入 $3 \mu \mathrm{mol}$ 卟啉溶液. 用微量注射器每隔 $5 \mathrm{~min}$ 各加入 $1 \mu \mathrm{L}$ 的 DNA, 使 DNA 与卟啉的浓度比值不断增加, 直 至饱和(总加入量为 $10 \mu \mathrm{L}$ ). 每次加 DNA 前检测其在 $300 \sim 600 \mathrm{~nm}$ 范围内的紫外-可见吸收光谱.

\section{2 结果与讨论}

\section{1 合成与表征}

四对桥连卟啉 5 12 均经缩合、配位、选择性硝化、 亲核取代和成醚等六步反应. 柱层析过程中, 第一主带 (卟啉 5 8) 前为少量未反应完的原料卟啉 $1 \sim 4$, 第二主 带为卟啉 9 12, 过量的和厚朴酚滞留于硅胶柱底带. 构型差异尤其是羟基的位置使同分异构体与硅胶结合 能力不同，卟啉 9 12 可能由于空间效应中羟基基团外 露而与硅胶结合更紧密, 在卟啉 $\mathbf{5} \sim 8$ 之后被洗脱. 同分 异构体间 $R_{\mathrm{f}}$ 值相差并不大, 系列化合物的羟基与硅胶的 结合会造成产物拖带, 需恰当调整淋洗剂中氯仿和环己 烷比例以提高分离效果.

四对目标产物 $5 \sim 12$ 的紫外-可见光谱均具有由卟
啉环共轭体系的 $\pi-\pi *$ * 跃迁产生的特征吸收谱带, 其中 Soret 带位于 $420 \mathrm{~nm}$ 左右, Q 带位于 $510 \sim 650 \mathrm{~nm}$ 之间, 金属卟啉与原卟啉相比, 中心离子的配位使得卟啉大环 对称性增加, $\mathrm{Q}$ 带吸收峰数目减少. 红外吸收光谱在 $3435 \mathrm{~cm}^{-1}$ 附近均有明显强而宽的 $\mathrm{O}-\mathrm{H}$ 伸缩振动特征 峰, $2925,2850 \mathrm{~cm}^{-1}$ 附近为卟啉中位苯环 $\mathrm{C}-\mathrm{H}$ 伸缩振 动所致, $1625 \sim 1465 \mathrm{~cm}^{-1}$ 归属为芳环骨架振动吸收峰, $1265,1170 \mathrm{~cm}^{-1}$ 两处中强峰为 $\mathrm{C}-\mathrm{O}-\mathrm{C}$ 和 $\mathrm{C}-\mathrm{O}$ 伸缩 振动, $800 \sim 700 \mathrm{~cm}^{-1}$ 范围三处尖峰为 $\mathrm{CH}_{2}$ 变形振动, 金 属卟啉中由于金属离子引起环的变形振动增强, 使得原 卟啉 $966 \mathrm{~cm}^{-1}$ 处 $\mathrm{N}-\mathrm{H}$ 弯曲振动峰被 $1005 \mathrm{~cm}^{-1}$ 附近的 金属-氮键吸收峰取代.

化合物 $\mathbf{6} \sim \mathbf{8}$ 和 $\mathbf{1 0} \sim \mathbf{1 2}$ 以 $\mathrm{CDCl}_{3}$ 为溶剂的 ${ }^{1} \mathrm{H} \mathrm{NMR}$ 图谱中, $\delta 8.89 \sim 8.37$ 归属为吡咯环上的氢 $(7 \mathrm{H}), \delta 8.28 \sim$ 7.39 归属为卟啉中位苯环上的氢 $(20 \mathrm{H}), \delta \quad 7.36 \sim 6.18$ 归 属为荎基和芳环氢所致 $(12 \mathrm{H}), \delta \quad 6.16 \sim 6.01$ 处单峰为羟 基氢 $(1 \mathrm{H}), \delta 5.93 \sim 4.72$ 为烯氢 $(6 \mathrm{H}), \delta 3.89 \sim 2.85,1.41 \sim$ $1.22,0.89 \sim 0.77$ 三处多重峰分别归属为亚甲基和碳链 $\alpha$, $\beta, \gamma$-位的氢 $(16 \mathrm{H}), \mathrm{NH}$ 质子化学位移出现在 $\delta-2.60$ $(2 \mathrm{H})$. 与紫外-可见光谱和红外光谱类似, 各同分异构 体一维核磁无明显区别, 根据二维核磁氢一氢相关谱 (COSY)中检测到的空间相近位置质子信号的分析, 卟 啉 $6 \sim 8$ 相比 10 $\sim 12$ 在碳链 $\alpha, \beta$-位的质子和 aryl- $\mathrm{CH}_{2} \mathrm{C}$ $\mathrm{H}=\mathrm{CH}_{2}$ 基团上的氢显示出更多偶合相关关系. 例如卟 啉 6 在 $(1.6,3.6)$ 和对应的 $(3.6,1.6)$ 处为碳链 $\beta$-位质子与 $\operatorname{aryl}-\mathrm{CH}_{2}$ 基团中亚甲基氢的偶合信号，(3.0,4.8)，(3.2， $5.0)$ 和对应的 $(4.8,3.0),(5.0,3.2)$ 处为碳链 $\alpha$-位质子与 $\mathrm{C}$ $=\mathrm{CH}_{2}$ 中烯氢的偶合信号, 而卟啉 $\mathbf{1 0}$ 由于碳链与 aryl- $\mathrm{CH}_{2} \mathrm{CH}=\mathrm{CH}_{2}$ 基团相隔更多的碳原子数, 空间位置 距离较远而没有相应的相关信号. H-H COSY 进一步确 证了同分异构体 ${ }^{1} \mathrm{H}$ NMR 谱图中各吸收峰位置的归属.

采用超高灵敏电喷雾法(QFT-ESI)对产物进行高分 辨质谱分析, 四对目标产物 $5 \sim 12$ 分别结合了 $\mathrm{H}^{+}, \mathrm{Na}^{+}$ 和 $\mathrm{K}^{+}$后均能在最高质量端给出相应分子离子峰基峰, 得到的谱图与预期一致, 卟啉 $5 \sim 8$ 结合 $\mathrm{Na}^{+}$时的丰度为 $100 \%$, 而相应的同分异构体卟啉 $\mathbf{9} \sim \mathbf{1 2}$ 在结合 $\mathrm{H}^{+}$时丰 度为 $100 \%$. 同时, 系列新化合物元素分析结果与目标 组成在误差范围内.

\section{2 卟啉产生单线态氧的能力}

卟啉对肿瘤的有效光损伤主要源于其在光照下产 生的单线态氧，单线态氧产率的高低即在一定程度决定 了卟啉作为光敏剂的潜在能力大小 ${ }^{[10]} ; 1,3-$ 二苯基异苯 并呋喃(DPBF) 是一种优良的单线态氧捕捉剂, 通过紫 外分光光度计测定卟啉不同光照时间过程中 DPBF 在 $415 \mathrm{~nm}$ 吸光度的变化, 可检测卟啉产生单线态氧的能 
力. 基于顺磁性金属离子铜可降低光敏反应中分子的三 线态寿命 ${ }^{[11]}$, 导致单线态氧产率降低, 故未对铜卟啉 $\mathbf{5}$ 和 9 进行测试. 图 1 中曲线的斜率即代表产生单线态氧 的能力, 卟啉 $6 \sim 8$ 和 $10 \sim 12$ 对应斜率依次为 -0.01756 , $-0.01598,-0.01754,-0.01838,-0.01986$ 和 0.01676 , 可见三对卟啉均有明显的产生单线态氧的能 力, 同分异构体之间产单线态能力略有不同, 但差距不 大.

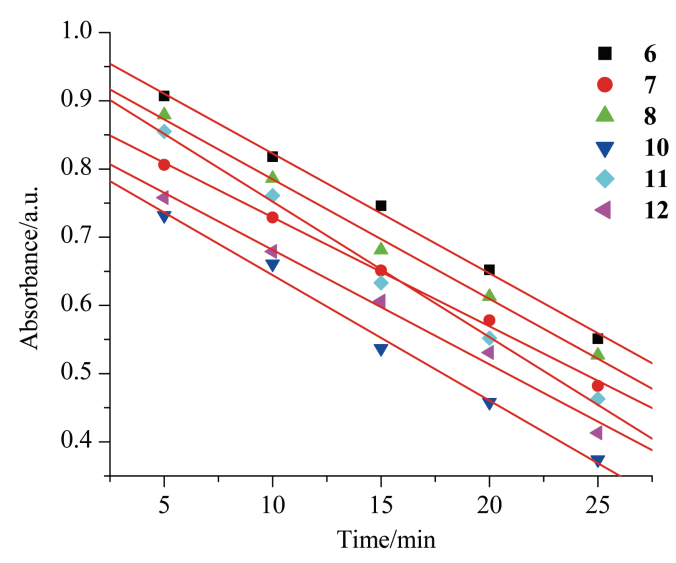

图 1 在卟啉 $6 \sim 8$ 和 $10 \sim 12$ 作用下 DPBF 的分解率

Figure 1 Decomposition of DPBF by porphyrins $\mathbf{6} \sim \mathbf{8}$ and $10 \sim 12$

\section{3 卟啉切割 pBR322 质粒 DNA}

分别对梯度浓度的卟㕲 5 12 在光照和无光照条件 进行 DNA 切割试验, 卟啉 $6 \sim 8$ 和 $10 \sim 12$ 光照下对超 螺旋 $\mathrm{pBR} 322$ 质粒 DNA 有良好切割, 金属铜卟啉 5 和 9 切割作用相对较弱, 无光照条件切割均不明显. 图 2 为 光照时卟啉 8 和 10 的 DNA 切割效果, 其中(Lane 1 Lane 4, Lane 6 Lane 9) 可见部分超螺旋 DNA (Form I) 转化为缺刻型 DNA (Form II), 显示出很低浓度的卟啉 在较短光照时间就能对质粒 DNA 进行切割作用, 且同 分异构体之间切割效果相近, 而单体卟啉切割作用不明 显. 表明切割过程中, 新型卟啉作为光敏剂在光照时产 生的单线态氧对 DNA 的光敏作用占主导, 与单线态氧

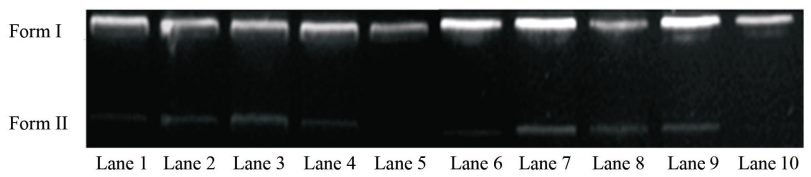

图 2 卟啉 $\mathbf{8}$ 和卟啉 $\mathbf{1 2}$ 对超螺旋 $\mathrm{pBR} 322$ 质粒的 DNA 切割 Figure 2 Cleavage of supercoiled pBR322 DNA by porphyrins $\mathbf{8}$ and $\mathbf{1 2}$

Concentration of porphyrin $\mathbf{8}+\mathrm{h} v(60 \mathrm{~min})+\mathrm{DNA}$, Lane 1: $40 \mu \mathrm{mol} / \mathrm{L}$; Lane 2: $30 \mu \mathrm{mol} / \mathrm{L}$; Lane 3: $20 \mu \mathrm{mol} / \mathrm{L}$; Lane 4: $10 \mu \mathrm{mol} / \mathrm{L}$; Lane 5: 0 $\mu \mathrm{mol} / \mathrm{L}$. Concentration of porphyrin $\mathbf{1 2}+\mathrm{h} v(60 \mathrm{~min})+\mathrm{DNA}$, Lane $6: 40$ $\mu \mathrm{mol} / \mathrm{L}$; Lane 7: $30 \mu \mathrm{mol} / \mathrm{L}$; Lane 8: $20 \mu \mathrm{mol} / \mathrm{L}$; Lane 9: $10 \mu \mathrm{mol} / \mathrm{L}$; Lane 10: $0 \mu \mathrm{mol} / \mathrm{L}$.
产率一致. 基于有效切割是建立在较好单线态氧产率及 分子与 DNA 的较好结合双重基础之上 ${ }^{[12]}$ ，进一步通过 与小牛胸腺 DNA 的相互作用初步探讨了桥连卟啉与 DNA 的作用能力和结合模式.

\section{4 卟啉与 CT DNA 相互作用}

紫外-可见光谱滴定法是研究卟啉与 DNA 结合能 力和作用模式的一种常用方法 ${ }^{[13]}$, 卟啉 5 12 与 CT DNA 相互作用后, Soret 带均表现明显减色而无红移现 象, 且卟啉 $5 \sim 8$ 减色更大. 图 3 为卟啉 7 和 11 与 CT DNA 的相互作用，其中卟啉 7 的 Soret 带减色约 33\%, 相同条件下卟啉 11 为 $27 \%$, 均大于单体卟啉的减色 $(<$ $20 \%$ ), 表明和厚朴酚的引入可能使卟啉 $5 \sim 12$ 更易与 DNA 结合，且卟啉 $\mathbf{5} \sim \mathbf{8}$ 相比 9 $\sim 12$ 其空间构型更具优 势. 根据 Pasternack 判据 ${ }^{[14]}$ 初步判断, 卟啉与 CT DNA 可能是自堆积的外部键合作用模式, 进一步研究有待通 过圆二色谱 $(\mathrm{CD})$ 等测试确证.
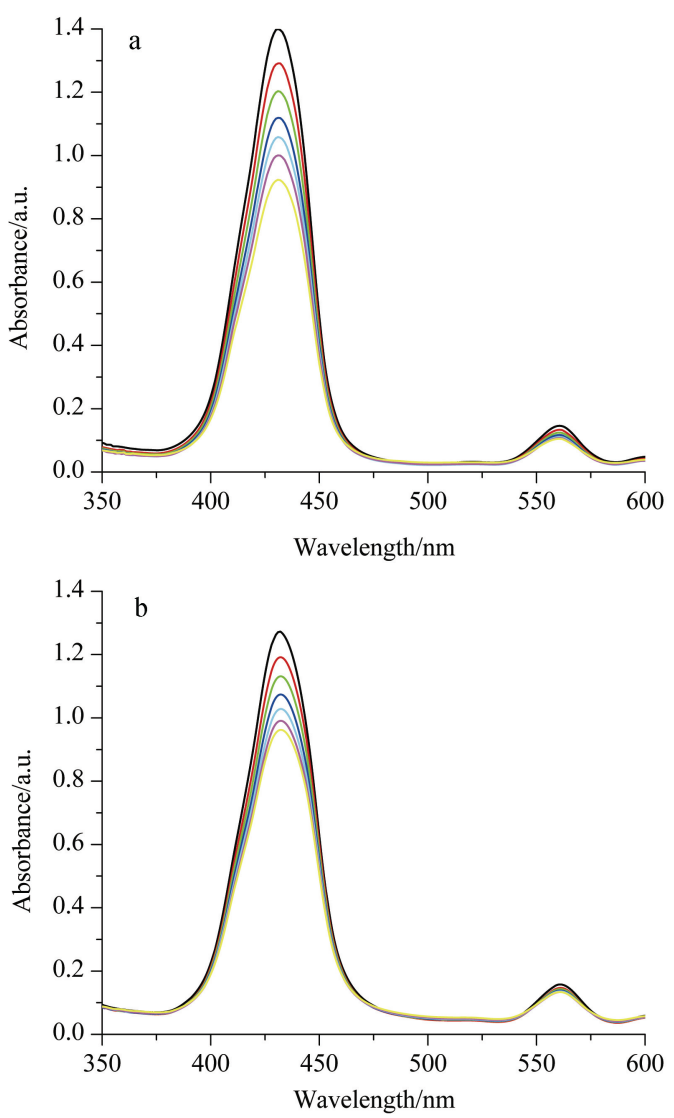

图 3 卟啉 7 (a)和卟啉 $\mathbf{1 1}$ (b)与 CT DNA 相互作用的紫外-可 见吸收光谱

Figure 3 UV-Vis absorbance of porphyrins 7 (a) and 11 (b) when titrated by CT DNA

\section{3 结论}

通过官能团修饰设计合成了四对兼具光敏性和抗 
癌性的天然产物桥连卟啉化合物, 通过多种分析方法对 目标产物进行了结构表征, 凝胶电泳试验表明, 新型光 敏药剂光照条件对 pBR322 质粒 DNA 有良好切割效果, 紫外-可见光谱滴定初步估计其与 CT DNA 的相互作用 为自堆积的外部键合作用模式, 具体作用模式有待深入 研究. 进一步将采用体外抗肿瘤细胞测试比较同分异构 体卟啉的光敏及抗癌活性, 同时研究探讨桥连基团和厚 朴酚在光敏剂毒性中的作用机理.

\section{References}

[1] Baptista, M. S.; Wainwright, M. Braz. J. Med. Biol. Res. 2011, 44, 1.

(b) Liu, Y.; Ma, X. Q.; Jin, P.; Li, H. T.; Zhang, R. R.; Ren, X. L.; Wang, H. B.; Tang, D. M.; Tian, W. R. Vet. J. 2011, 188, 325.

(c) Threes, G. M. S.; Stan, P. Photochem. Photobiol. 2011, 87, 2.

[2] (a) Gelfuso, G. M.; Gratieri, T.; Souza, J. G.; Thomazine, J. A.; Lopez, R. F. V. Eur. J. Pharm. Biopharm. 2011, 77, 249.

(b) Ethirajan, M.; Chen, Y.; Joshi, P.; Pandey, R. K. Chem. Soc. Rev. 2011, 40, 340.

[3] Eriksson, E. S. E.; Eriksson, L. A. Phys. Chem. Chem. Phys. 2011, 13, 7207 .

[4] (a) Ishizuka, M.; Abe, F.; Sano, Y.; Takahashi, K.; Inoue, K.; Nakajima, M.; Kohda, T.; Komatsu, N. Int. Immunopharmacol. 2011, 11,358 .

(b) Angela, C.; Ilaria, P.; Giuseppe, P. Cancers 2011, 3, 1014.

[5] (a) Smith, K.; Malatesti, N.; Cauchon, N.; Hunting, D.; Lecomte, R.; Lier, J. E.; Greenman, J.; Boyle, R. W. Immunology 2011, 132, 256.

(b) Ruthard, C.; Schmidt, M.; Groehn, F. Macromol. Rapid Commun. 2011, 32, 706.

[6] (a) Tang, X. J.; Yao, K.; Zhang, L. Eur. J. Pharmacol. 2011, 650, 72 .

(b) Wu, J. P.; Zhang, W.; Wu, F. Inflammation Res. 2010, 59, 1073.

[7] Huang, Q. M.; Pan, Z. Q.; Wang, P.; Chen, Z. P.; Zhang, X. L.; Xu, H. S. Bioorg. Med. Chem. Lett. 2006, 16, 3030.
[8] Zheng, Y. M.; Wang, K.; Li, T.; Zhang, X. L.; Li, Z. Y. Molecules 2011, 16, 3488.

[9] (a) Huang, Q. M.; Pan, W.; Li, Q.; Wang, S. W.; Li, Z. Y.; Pan, Z. Q. Chin.J. Org. Chem. 2011, 31, 336 (in Chinese).

(黄齐茂, 潘威, 李清, 王司卫, 李志远, 潘志权, 有机化学, 2011, 31, 336.)

(b) Huang, Q. M.; Li, Z. Y.; Xiao, X.; Pan, Z. Q.; Deng, P. X. Chin. J. Org. Chem. 2010, 30, 79 (in Chinese).

(黄齐茂, 李志远, 肖欣, 潘志权, 邓鹏星, 有机化学, 2010, 30, 79.)

(c) Huang, Q. M.; Deng, P. X.; Li, Z. Y.; Zhou, H.; Hu, X. L.; Pan, Z. Q. Acta. Chim. Sinica 2010, 68, 1070 (in Chinese).

(黄齐茂, 邓鹏星, 李志远, 周红, 胡学雷, 潘志权, 化学学报, 2010, 68, 1070.)

(d) Huang, Q. M.; Chen, Z. P.; Xu, H. S.; Wu, X. J.; Zhao, Y. G.; Zhang, X. W. Chin. J. Org. Chem. 2001, 21, 746 (in Chinese).

(黄齐茂, 陈彰评, 徐汉生, 吴萱阶, 赵勇刚, 张小伟, 有机化学, 2001, 21, 746.)

[10] (a) Choi, K. H.; Wang, K. K., Shin, E. P.; Oh, S. L.; Jung, J. S.; Kim, H. K.; Kim, R. Y. J. Phys. Chem. C 2011, 115, 3212.

(b) Ringot, C.; Sol, V.; Barriere, M.; Saad, N.; Bressollier, P.; Granet, R.; Couleaud, P.; Frochot, C.; Krausz, P. Biomacromolecules 2011, 12, 1716.

[11] (a) Zhao, P.; Huang, J. W.; Ji, L. N. J. Coord. Chem. 2011, 64, 1977.

(b) Pineiro, M.; Carvalho, A. L.; Pereira, M. M.; Gonsalves, A. M.; Arnaut, L. G.; Formosinho, S. J. Chem. Eur. J. 1998, 4, 2299.

[12] (a) Kee, T. W.; Adhikary, R.; Carlson, P. J.; Mukherjee, P.; Petrich, J. W. Aust. J. Chem. 2011, 64, 23.

(b) Filip, A. G.; Clichici, S.; Daicoviciu, D.; Ion, R. M.; Tatomir, C.; Rogojan, L.; Opris, I.; Mocan, T. Braz. J. Med. Biol. Res. 2011, 44, 53.

[13] (a) Brewer, A.; Siligardi, G.; Neylon, C.; Stulz, E. Org. Biomol. Chem. 2011, 9, 777.

(b) Ananyan, G.; Avetisyan, A.; Aloyan, L.; Dalyan, Y. Biophys. Chem. 2011, 156, 96.

[14] Pasternack, R. F.; Gibbs, E. J.; Vilafranca, J. J. Biochemistry 1983, 22,5409 . 